\title{
Dimensionamento de redes de distribuição de água por meio de análise multicriterial
}

Data de entrada: $26 / 03 / 2018$

\section{Multicriterial water distribution networks design}

Data de aprovação: $27 / 06 / 2018$

Gustavo Meirelles Lima ${ }^{1 * *}$ | Bruno Melo Brentan ${ }^{1}$ | Antonio Carlos Zuffo | Edevar Luvizotto Junior² ～DOI: https://doi.org/10.36659/dae.2020.010

ORCID ID

Meirelles G (D) https://orcid.org/0000-0002-1971-3970

Brentan B.M (D) https://orcid.org/0000-0003-0616-2281
Zuffo A.C (D) https://orcid.org/0000-0002-2186-9755

Luvizzoto JrE (D) https://orcid.org/0000-0002-4606-4996

\section{Resumo}

O dimensionamento de redes de distribuição de água (RDAs) é feito para que as restrições operacionais de velocidade e pressão se mantenham dentro de limites que garantam a eficiência operacional. Assim, buscam-se as tubulações de menor custo para que essas condições sejam satisfeitas. Entretanto, ao adotar essa prática, outras características da rede podem ser afetadas negativamente, como sua resiliência e capacidade de expansão. Dessa forma, este trabalho apresenta um estudo de caso em que a análise multicriterial é utilizada para realizar o dimensionamento de uma RDA. Primeiramente, um mapa cognitivo é feito para identificar os principais critérios a serem considerados na solução do problema. Em seguida, o método Delphi é usado em conjunto com o Analytic Hierarchy Process (AHP) para determinar os pesos relativos de cada critério. Por fim, três diferentes métodos de análise multicritérios são utilizados para a solução do problema: AHP, Electre e Promethee.

Palavras-chave: Rede de distribuição. Otimização. Análise multicritério.

\section{Abstract}

The design of water distribution networks (WDNs) is made to match velocity and pressure constraints that guarantee operational efficiency. Thus, pipes with lower cost are selected to attend these conditions. However, this procedure can harm other characteristics of the network, as for example its resilience and expansion capacity. Therefore, this paper presents a case study in which the multicriterial analysis is used to design a WDN. First, a cognitive map is built to identify the main criteria to be considered during the design process. Then, the Delphi method is used jointly with the Analytic Hierarchy Process (AHP) to define the relative weights of each criteria. Finally, three different methods for multicriterial analysis are used to solve the problem: AHP, Electre and Promethee.

Keywords: Water distribution network. Optimization. Multicriterial analysis.

\footnotetext{
${ }^{1}$ UFMG - Universidade Federal de Minas Gerais. Escola de Engenharia - Depto. Eng. Hidráulica e Recursos Hídricos. Av. Antônio Carlos, 6627 Pampulha, CEP 31270-901 - Belo Horizonte (MG), Brasil.

${ }^{2}$ Unicamp (Universidade Estadual de Campinas)/FEC - Faculdade de Engenharia Civil - Departamento de Recursos Hídricos . Campinas (SP), Brasil.

* Autor correspondente: gustavo.meirellesaehr.ufmg.br.
} 


\section{INTRODUÇÃO}

As redes de distribuição de água (RDAs) constituem a maior parte do investimento inicial das companhias de saneamento. Além das tubulações, outros dispositivos, como válvulas, bombas e reservatórios são fundamentais para a boa operação do sistema, atendendo aos consumidores em quantidade e qualidade, ou seja, dentro de limites normativos de pressão e velocidade de escoamento (SWAMME E SHARMA, 2008). Por se tratar do principal ativo das empresas, Gomes (2011) ressalta a busca pelo menor custo de implantação em um projeto. Entretanto, além dos custos de implantação, os custos operacionais também devem ser considerados nessa busca pelo melhor projeto. Assim, os gastos com energia elétrica em estações elevatórias também devem ser adicionados (DANDY et al., 1994). Recentemente, Meirelles et al. (2017) também propuseram o dimensionamento considerando a geração de energia elétrica, sendo neste caso descontado o benefício obtido com a produção de energia ao longo da vida útil do custo total de implantação.

Para solucionar o problema de dimensionamento de RDAs, diversos trabalhos aplicam técnicas de otimização meta-heurísticas mono-objetivas acopladas a simuladores hidráulicos para obter a configuração capaz de atender aos critérios normativos com o menor custo de implantação (SIMPSON et al., 1993; CUNHA E SOUZA, 2001; MOOSAVIAN E ROODSARI, 2014). Apesar da ampla utilização, esses métodos não garantem a obtenção da solução ótima global. Além disso, Maier (2015) ressalta que a maioria dos trabalhos é feito em redes muito simples, que não condizem com a realidade, enquanto Marchiori et al. (2017) demonstram que as funções de penalização, utilizadas para o tratamento das restrições nos métodos meta-heurísticos, também são um fator de incerteza na busca pela melhor solução.
Devido a essas incertezas, o uso de técnicas multi-objetivo tem ganhado destaque para a solução desse problema (MONTALVO et al., 2014). Essas técnicas utilizam não apenas o custo como referência no processo de dimensionamento, mas também outros indicadores, como a resiliência do sistema (OSTFELD, 2013) e a qualidade da água (FARMANI et al., 2006).

Dessa forma, neste trabalho será avaliada a performance do dimensionamento de uma RDA utilizando métodos multicriteriais para a tomada de decisão. Após a elaboração do mapa cognitivo do problema, oito diferentes critérios serão considerados para realizar o dimensionamento da rede: resiliência, qualidade da água, custo operacional, custo de implantação, pressão disponível, velocidade de escoamento, satisfação da população e impactos ambientais. Os pesos de cada um desses critérios é obtido por meio do método Delphi em conjunto com o Analytic Hierarchy Process (AHP). Em seguida, três diferentes métodos de análise multicriterial, AHP, Electre e Promethee são utilizados para avaliar seis diferentes alternativas de dimensionamento da rede Two-Loop (ALPEROVITS E SHAMIR, 1977).

\section{METODOLOGIA}

\subsection{Mapa Cognitivo}

Os mapas cognitivos são representações gráficas resultantes da interpretação de determinado problema. De acordo com Ackermann et al. (2004), eles podem ser utilizados para estruturar, analisar e dar sentido aos problemas. Para sua elaboração, Ribeiro et al. (2011) destacam a importância da opinião de especialistas no problema em questão. Assim, após definir como rótulo do problema "Dimensionamento de Redes de Abastecimento de Água", os integrantes do Laboratório de Hidráulica Computacional (LHC) da Universidade Estadual de Campinas (Unicamp) 
foram questionados sobre quais seriam os parâmetros mais importantes a serem avaliados nesse problema. As respostas obtidas foram organizadas no mapa cognitivo apresentado na Fig. 1, levando em consideração os questionamentos fundamentais: "Por que este parâmetro é importante?" e "Como posso obter o resultado positi- vo?". Analisando o mapa cognitivo, não é possível identificar claramente clusters ou linhas de argumentação, devido à alta interrelação entre os parâmetros. Ainda assim, é nítida a formação de quatro conceitos-fim fundamentais: satisfação da população, impactos ambientais e custos de implantação e operacionais.

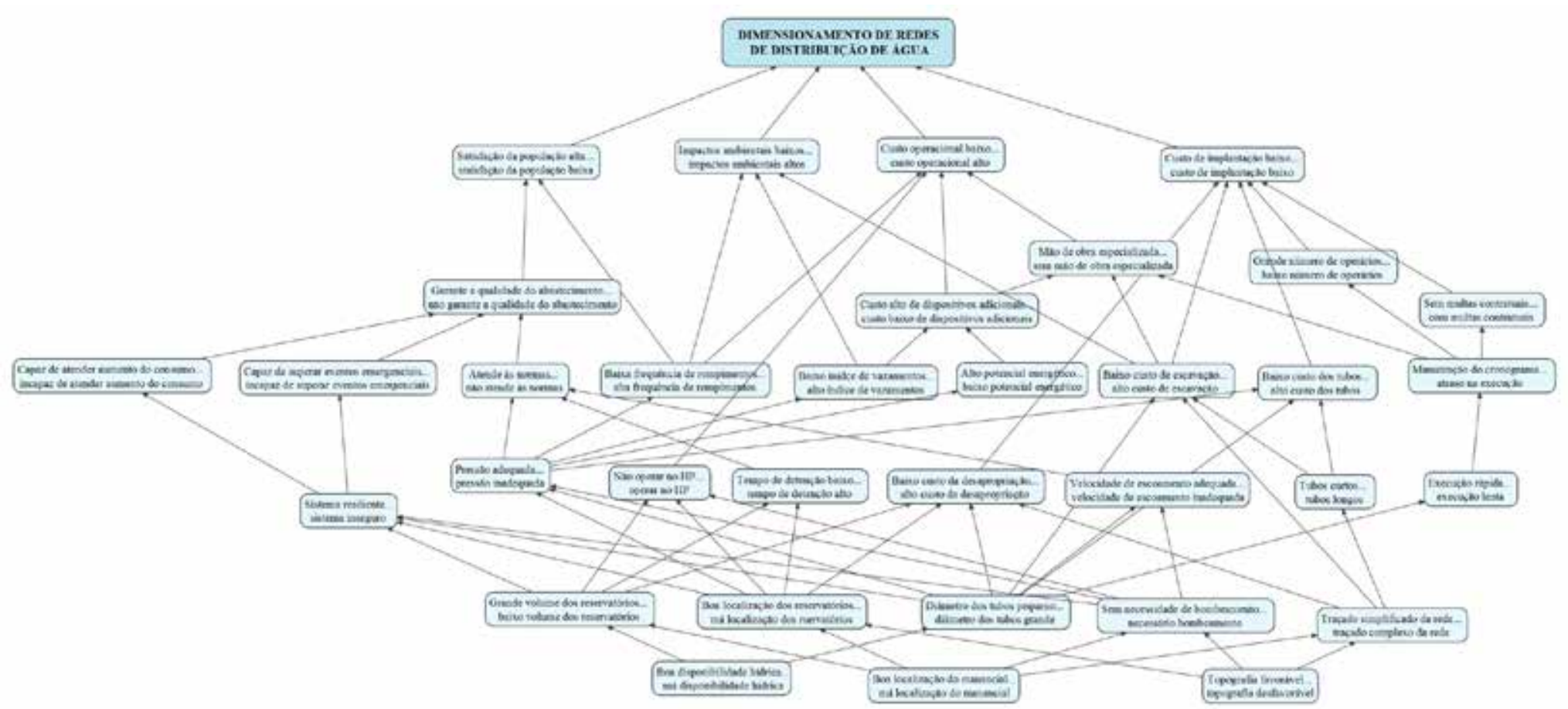

Figura 1 - Mapa cognitivo para dimensionamento de redes de distribuição de água 


\subsection{Critérios e Pesos}

Com base nos resultados obtidos com o mapeamento cognitivo, oito critérios de avaliação foram selecionados para realizar a análise multicritério, conforme ilustra a Fig. 2.

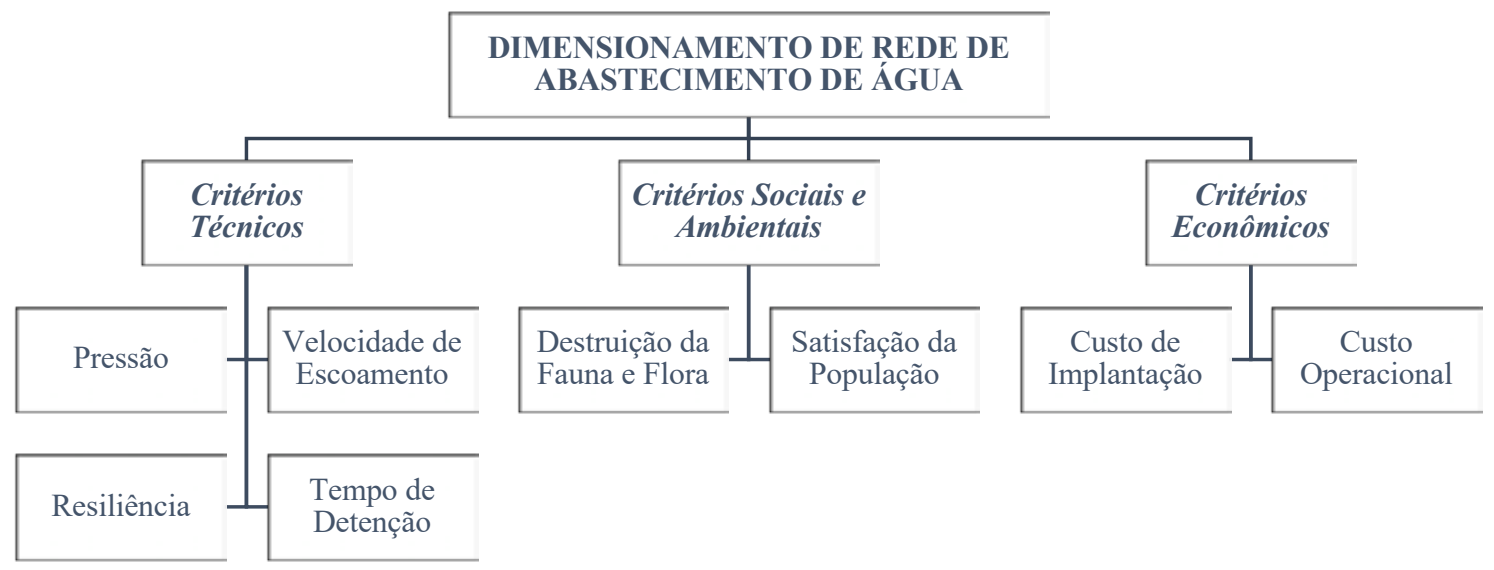

Figura 2 - Representação hierárquica dos critérios utilizados

Para obter a importância relativa de cada um dos critérios e seus respectivos pesos na avaliação multicritério, foi utilizado método Delphi (DALKEY E HEMER, 1963), em que os integrantes do grupo LHC/Unicamp foram questionados sobre a importância de cada um dos critérios escolhidos, atribuindo notas de 1 a 10 . O método AHP foi utilizado em conjunto, pois permite a comparação entre pares de critério para que sejam definidas as respectivas importâncias relativas (BRIOZO E MUSETTI, 2015).
Assim, após uma primeira rodada de avaliação dos critérios, foram extraídos os valores referentes ao primeiro e terceiro quartis da amostra. Os valores fora dessa região são considerados anormais, e o questionário foi reenviado ao grupo, perguntando aos avaliadores se eles desejavam alterar a nota dos critérios marcados. Após essa segunda rodada, novamente foram encontrados valores fora da região de interesse. Esses valores foram desconsiderados, e a média de cada um dos critérios foi calculada. A Tabela 1 resume o processo de obtenção das notas dos critérios.

Tabela 1 - Resultados obtidos com os questionários

\begin{tabular}{|c|c|c|c|c|c|c|c|c|c|c|c|c|}
\hline \multirow{2}{*}{ Critério/Especialista } & \multicolumn{10}{|c|}{ PRIMEIRA RODADA } \\
\cline { 2 - 11 } & A & B & C & D & E & F & G & H & I & J & K & L \\
\hline Pressão & 7 & 5 & 7 & 9 & 8 & 7 & 7 & 8 & 8 & 7 & 9 & 7 \\
\hline Velocidade & 6 & 5 & 7 & 8 & 7 & 7 & 6 & 8 & 7 & 7 & 8 & 8 \\
\hline Tempo de detenção & 7 & 5 & 9 & 7 & 8 & 9 & 7 & 8 & 8 & 6 & 9 & 8 \\
\hline Resiliência & 5 & 5 & 8 & 8 & 6 & 7 & 7 & 6 & 7 & 7 & 7 & 7 \\
\hline Satisfação da população & 8 & 9 & 8 & 8 & 6 & 9 & 9 & 8 & 6 & 7 & 7 & 8 \\
\hline Impactos ambientais & 7 & 9 & 8 & 7 & 8 & 9 & 8 & 7 & 6 & 7 & 9 & 8 \\
\hline Custo operacional & 10 & 8 & 8 & 9 & 10 & 8 & 8 & 9 & 9 & 9 & 10 & 9 \\
\hline Custo de implantação & 9 & 8 & 9 & 9 & 8 & 7 & 8 & 8 & 9 & 8 & 10 & 9 \\
\hline
\end{tabular}

\begin{tabular}{|c|c|}
\hline $\begin{array}{c}\text { Primeiro } \\
\text { Quartil }\end{array}$ & $\begin{array}{c}\text { Terceiro } \\
\text { Quartil }\end{array}$ \\
\hline 7.0 & 8.0 \\
\hline 6.8 & 8.0 \\
\hline 7.0 & 8.3 \\
\hline 6.0 & 7.0 \\
\hline 7.0 & 8.3 \\
\hline 7.0 & 8.3 \\
\hline 8.0 & 9.3 \\
\hline 8.0 & 9.0 \\
\hline
\end{tabular}

\begin{tabular}{|c|c|c|c|c|c|c|c|c|c|c|c|}
\hline \multicolumn{10}{|c|}{ SEGUNDA RODADA } \\
\hline A & B & C & D & E & F & G & H & I & J & K & L \\
\hline 7 & 6 & 7 & 8 & 8 & 7 & 7 & 8 & 8 & 7 & 9 & 7 \\
\hline 7 & 6 & 7 & 8 & 7 & 7 & 6 & 8 & 7 & 7 & 8 & 8 \\
\hline 7 & 6 & 9 & 7 & 8 & 9 & 7 & 8 & 8 & 7 & 9 & 8 \\
\hline 5 & 6 & 8 & 8 & 6 & 7 & 7 & 6 & 7 & 7 & 7 & 7 \\
\hline 8 & 9 & 8 & 8 & 7 & 9 & 9 & 8 & 7 & 7 & 7 & 8 \\
\hline 7 & 9 & 8 & 7 & 8 & 9 & 8 & 7 & 7 & 7 & 9 & 8 \\
\hline 10 & 8 & 8 & 9 & 9 & 8 & 8 & 9 & 9 & 9 & 10 & 9 \\
\hline 9 & 8 & 9 & 9 & 8 & 8 & 8 & 8 & 9 & 8 & 9 & 9 \\
\hline 7.6 \\
\hline 7.8 \\
\hline 7.8 \\
\hline 8.8 \\
\hline
\end{tabular}


Os resultados mostram que o custo operacional é o parâmetro mais importante para o dimensionamento de uma RDA, enquanto a resiliência se mostrou o critério menos relevante no projeto. Em seguida, o método AHP foi aplicado para a obtenção dos pesos dos parâmetros. Primeiramente, os critérios foram ordenados de forma decrescente em seu grau de importância. Com isso, uma matriz de diferenças entre as importâncias foi obtida (Tabela 3). Assim, os valores calculados são relacionados com a escala proposta por Saaty (1991), conforme mostra a Tabela 2, resultando na segunda matriz de importância relativa apresentada na Tabela 3.

Tabela 2 - Definição da intensidade de importância dos critérios

\begin{tabular}{|c|c|c|}
\hline Grau de Importância & Definição & Diferença Máxima \\
\hline 1 & Mesma importância & 0 \\
\hline 2 & Intermediário entre 1 e 3 & 0.2 \\
\hline 3 & Importância pequena & 0.3 \\
\hline 4 & Intermediário entre 3 e 5 & 0.6 \\
\hline 5 & Importância grande ou essencial & 0.8 \\
\hline 6 & Intermediário entre 5 e 7 & 1.1 \\
\hline 7 & Importância muito grande ou demonstrada & 1.6 \\
\hline 8 & Intermediário entre 7 e 9 & 2.1 \\
\hline 9 & Importância absoluta & 2.5 \\
\hline
\end{tabular}

Tabela 3 - Matriz de comparação e de importância dos critérios

\begin{tabular}{|c|c|c|c|c|c|c|c|c|}
\hline Critério & 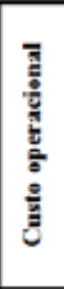 & 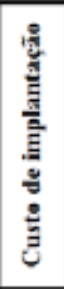 & 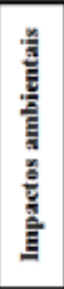 & 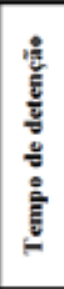 & 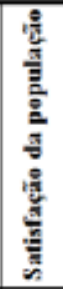 & 产 & $\begin{array}{l}\frac{0}{E} \\
\frac{\mathrm{E}}{\mathrm{E}} \\
\frac{\mathrm{c}}{\mathrm{y}}\end{array}$ & $\frac{a}{c}$ \\
\hline Custo operacional & 0.00 & 0.33 & 1.03 & 1.06 & 1.06 & 1.23 & 1.58 & 2.06 \\
\hline Custo de implantação & & 0.00 & 0.70 & 0.72 & 0.72 & 0.90 & 1.25 & 1.72 \\
\hline Impactos ambientais & & & 0.00 & 0.02 & 0.02 & 0.20 & 0.55 & 1.02 \\
\hline Tempo de detençào & & & & 0.00 & 0.00 & 0.18 & 0.53 & 1.00 \\
\hline Satisfação da população & & & & & 0.00 & 0.18 & 0.53 & 1.00 \\
\hline Pressão & & & & & & 0.00 & 0.35 & 0.82 \\
\hline Velocidade & & & & & & & 0.00 & 0.47 \\
\hline Resiliência & & & & & & & & 0.00 \\
\hline
\end{tabular}

\begin{tabular}{|c|c|c|c|c|c|c|c|}
\hline 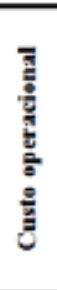 & 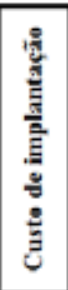 & 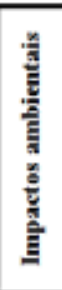 & 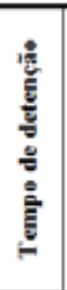 & 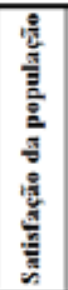 & 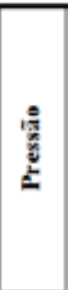 & $\begin{array}{l}\text { हैँ } \\
\text { हूँ } \\
\text { है }\end{array}$ & 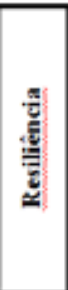 \\
\hline 1.00 & 4.00 & 6.00 & 6.00 & 6.00 & 7.00 & 7.00 & 8.00 \\
\hline 0.25 & 1.00 & 5.00 & 5.00 & 5.00 & 6.00 & 7.00 & 8.00 \\
\hline 0.17 & 0.20 & 1.00 & 2.00 & 2.00 & 2.00 & 4.00 & 6.00 \\
\hline 0.17 & 0.20 & 0.50 & 1.00 & 1.00 & 2.00 & 4.00 & 6.00 \\
\hline 0.17 & \begin{tabular}{|l}
0.20 \\
\end{tabular} & 0.50 & 1.00 & 1.00 & 2.00 & 4.00 & 6.00 \\
\hline 0.14 & \begin{tabular}{|l|}
0.17 \\
\end{tabular} & 0.50 & 0.50 & 0.50 & 1.00 & 4.00 & 6.00 \\
\hline 0.14 & 0.14 & 0.25 & 0.25 & 0.25 & 0.25 & 1.00 & 4.00 \\
\hline 0.13 & \begin{tabular}{|l|l|}
0.13 \\
\end{tabular} & 0.17 & 0.17 & 0.17 & 0.17 & 0.25 & 1.00 \\
\hline
\end{tabular}


Neste estágio, é preciso determinar o índice de consistência $C l$ e a razão de consistência $C R$, através das Eq. 1 e 2 respectivamente. Ambos os indicadores avaliam a consistência das comparações feitas, sendo que o $C R$ ainda considera um erro aleatório relacionado à ordem da matriz de importância relativa.

$C I=\frac{\lambda_{m}-n}{n-1}$

$C R=\frac{C I}{R I}$ em que $\mathrm{Cl}$ é o índice de consistência, $\lambda \mathrm{m}$ é a média dos autovalores da matriz de importância relativa, $n$ é a ordem da matriz de importância relativa, $C R$ é a razão de consistência e $R l$ é o índice de consistência aleatória $(R I=1.41$ para $n=8)$.

$O$ valor de $C R$ obtido é de 0.094 , indicando a consistência das comparações $(C R<0.1)$. Assim, os pesos apresentados na Tabela 4 podem ser utilizados na avaliação multicritério.

Tabela 4 - Pesos dos critérios adotados

\begin{tabular}{|c|c|c|c|}
\hline Critério & Peso & Critério & Peso \\
\hline Custo operacional & 3.73 & Satisfação da população & 0.69 \\
\hline Custo de implantação & 2.39 & Pressão & 0.54 \\
\hline Impactos ambientais & 0.90 & Velocidade & 0.28 \\
\hline Tempo de detenção & 0.69 & Resiliência & 0.16 \\
\hline
\end{tabular}

\subsection{Funções de Valor}

Para que as alternativas sejam avaliadas de acordo com os pesos encontrados, é necessário avaliar cada um dos critérios individualmente para cada uma das alternativas disponíveis. Assim, oito di- ferentes funções de valor foram criadas, conforme mostra a Fig. 3. Para cada uma das alternativas estudadas, uma simulação no software Epanet (Rossman, 2000) foi realizada para obter os valores de pressão, vazão e tempo de detenção. 


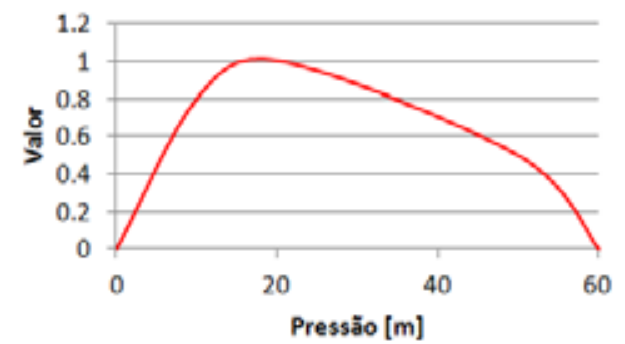

(a)

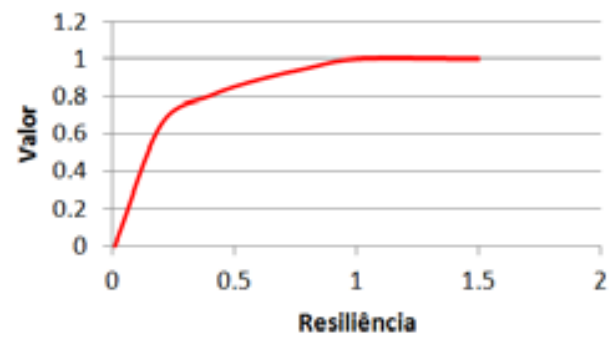

(c)

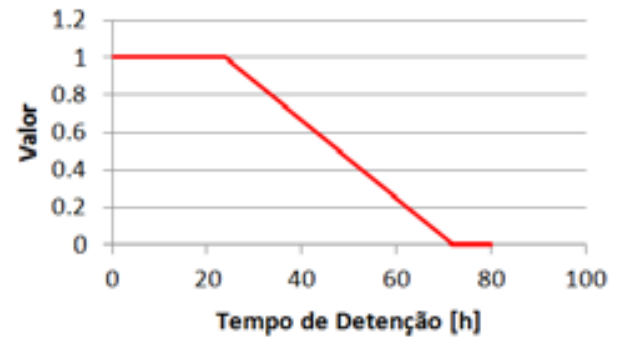

(e)

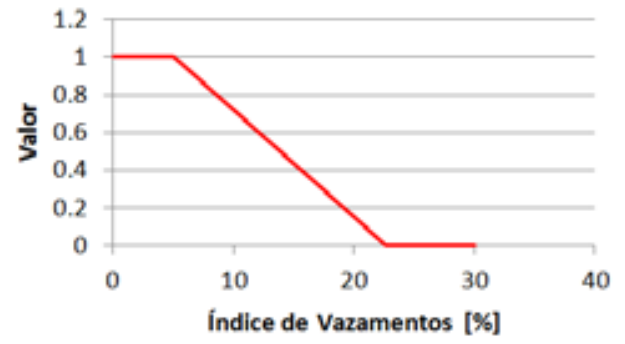

(g)

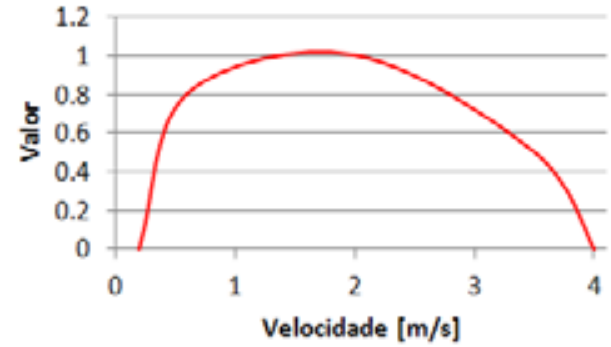

(b)

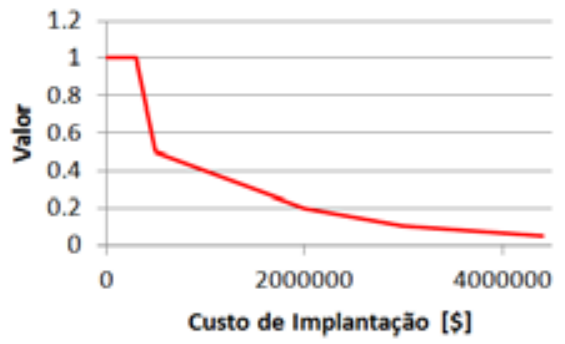

(d)

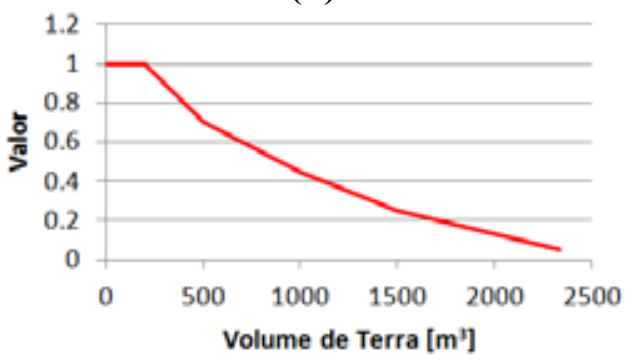

(f)

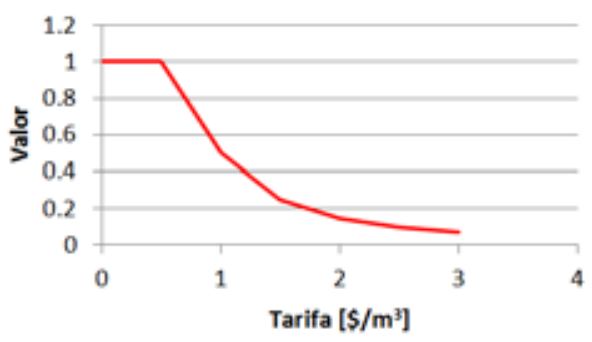

(k)

Figura 3 - Funções de valor: a) Pressão; b) Velocidade; c) Resiliência; d) Custo de implantação; e) Tempo de detenção; f) Impactos ambientais; g) Custo operacional; k) Satisfação da população 
As funções de valor de pressão e velocidade são restritas pelos limites operacionais estabelecidos pela NBR 12218 (ABNT, 1994), onde em que se observa-se uma queda acentuada dos valores dos critérios. Ainda que qualquer valor dentro dos limites estabelecidos sejam aceitáveisaceitável, considerou-se como ótima uma pressão de $20 \mathrm{~m}$ e uma velocidade de $2 \mathrm{~m} / \mathrm{s}$.

Os valores referentes ao impacto ambiental e ao custo operacional foram obtidos indiretamente calculando-se o volume de terra movimentado e o índice de vazamentos respectivamente, pois estes esses parâmetros estão diretamente relacionados à a estes esses critérios. Para a qualidade da água, considerou-se uma queda linear do seu valor para tempos de detenção entre 24 e $72 \mathrm{~h}$ de acordo com recomendação da American Water Works Association (AWWA, 2002). Para determinar a satisfação da população, utilizouse como referência a tarifa de água. Apesar de sua dependência de alguns dos critérios utilizados, ela possui uma forte influência política e climática, e portanto, seu valor foi determinado aleatoriamente dentro dos limites de sua função. Por fim, os custos de implantação e a resiliência (TODINI, 2000) do sistema são calculados a partir das Eq. 3 e 4 respectivamente.

$C T=\sum_{i=1}^{N T} C\left(D_{i}\right) \cdot L_{i}$

$$
I_{r}=\frac{\sum_{i=1}^{N N} q_{i} \cdot\left(h_{i}-h_{r e q_{i}}\right)}{\left(\sum_{k=1}^{N R} Q_{k} \cdot H_{k}\right)-\sum_{i=1}^{N N} q_{i} \cdot h_{r e q_{i}}}
$$

em que $C T$ é o custo de implantação, NT o número de tubos da rede, $C(D)$ o custo unitário de uma tubulação de diâmetro $D, L$ o comprimento da tubulação, $I_{r}$ o índice de resiliência, $N N$ o número de nós da rede, $q$ a demanda nodal, $h_{i}$ a carga piezométrica do nó $i, h_{\text {reqi }}$ a carga piezométrica mínima no nó $i, N R$ o número de reservatórios da rede, $Q_{k}$ a vazão que sai do reservatório $k$ e $H_{k}$ o nível d'água do reservatório $k$.

\section{ESTUDO DE CASO}

\subsection{Rede Two-Loop}

A rede conhecida como Two-loop foi proposta por Alperovits e Shamir (1977), e utilizada por diversos pesquisadores, consistindo em um importante benchmarking do problema de dimensionamento de redes. É composta por oito trechos em duas malhas, com seis nós de consumo abastecidos por um reservatório de nível fixo, conforme ilustra a Fig. 4. Em cada um dos nós foi adicionado um emissor para que os vazamentos sejam simulados, conforme estabelece a Eq. 5.

$q_{l}=p_{n}^{\beta}$

em que $q_{l}$ é a vazão do vazamento, $p_{n}$ a pressão do nó $n$ e $\beta$ o coeficiente do emissor. 


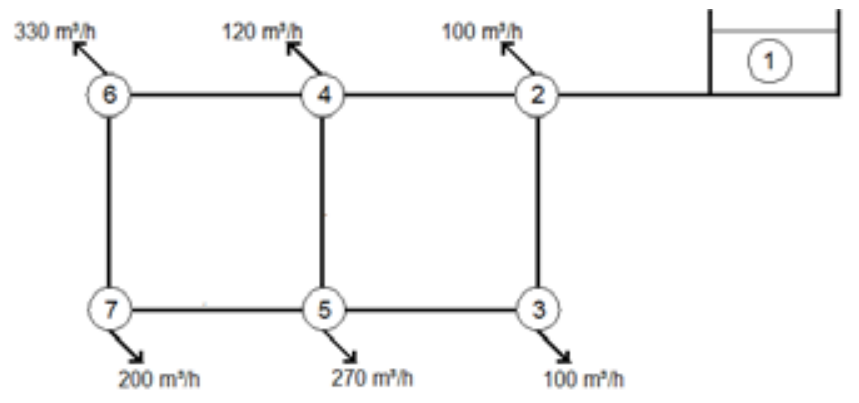

Figura 4 - Rede Two-loop

Os diâmetros disponíveis para implantação da rede são apresentados na Tabela 5, bem como seus custo unitários.

Tabela 5 - Tubulações disponíveis para implantação da rede e seus custo unitários

\begin{tabular}{|c|c|c|c|}
\hline Diâmetro [mm] & Custo Unitário $[\mathbf{\$} / \mathbf{m}]$ & Diâmetro $[\mathbf{m m}]$ & Custo Unitário $[\mathbf{\$} / \mathbf{m}]$ \\
\hline 25.4 & 2 & 304.8 & 50 \\
\hline 50.8 & 5 & 355.6 & 60 \\
\hline 76.2 & 8 & 406.4 & 90 \\
\hline 101.6 & 11 & 457.2 & 130 \\
\hline 152.4 & 16 & 508.0 & 170 \\
\hline 203.2 & 23 & 558.8 & 300 \\
\hline 254.0 & 32 & 609.6 & 550 \\
\hline
\end{tabular}

\subsection{Resultados e Discussão}

De acordo com a disponibilidade de tubulações, foram criadas seis alternativas distintas, sendo uma delas apenas com os diâmetros mínimos, outra apenas com os diâmetros máximos e quatro situações intermediárias, conforme mostra a Tabela 6.

Tabela 6 - Diâmetros das tubulações para cada uma das alternativas estudadas

\begin{tabular}{|c|c|c|c|c|c|c|c|c|}
\hline Alternativa / Tubulação & $\mathbf{1}$ & $\mathbf{2}$ & $\mathbf{3}$ & $\mathbf{4}$ & $\mathbf{5}$ & $\mathbf{6}$ & $\mathbf{7}$ & $\mathbf{8}$ \\
\hline $\mathbf{1}$ & 25.4 & 25.4 & 25.4 & 25.4 & 25.4 & 25.4 & 25.4 & 25.4 \\
\hline $\mathbf{2}$ & 508.0 & 254.0 & 304.8 & 50.8 & 355.6 & 254.0 & 254.0 & 25.4 \\
\hline $\mathbf{3}$ & 406.4 & 406.4 & 254.0 & 25.4 & 254.0 & 254.0 & 355.6 & 355.6 \\
\hline $\mathbf{4}$ & 406.4 & 254.0 & 355.6 & 254.0 & 304.8 & 25.4 & 254.0 & 304.8 \\
\hline $\mathbf{5}$ & 457.2 & 355.6 & 304.8 & 25.4 & 254.0 & 25.4 & 304.8 & 254.0 \\
\hline $\mathbf{6}$ & 609.6 & 609.6 & 609.6 & 609.6 & 609.6 & 609.6 & 609.6 & 609.6 \\
\hline
\end{tabular}

Com essas configurações, obtiveram-se os valores apresentados na Tabela 7 para cada um dos critérios avaliados. 
Tabela 7 - Valores dos critérios para cada uma das alternativas estudadas

\begin{tabular}{|c|c|c|c|c|c|c|c|}
\hline Alternativa / Critério & $\mathbf{1}$ & $\mathbf{2}$ & $\mathbf{3}$ & $\mathbf{4}$ & $\mathbf{5}$ & $\mathbf{6}$ \\
\hline Pressão & $-3.4 \mathrm{E}+07$ & 0.31 & 0.66 & 0.66 & 0.46 & 0.16 \\
\hline Velocidade & -285.13 & 0.10 & 0.53 & 0.23 & 0.18 & 0.08 \\
\hline Resiliência & 0.00 & 0.58 & 0.59 & 0.56 & 0.63 & 0.81 \\
\hline Tempo de detenção & 1.00 & 1.00 & 1.00 & 1.00 & 1.00 & 1.00 \\
\hline Impactos ambientais (volume escavado) & 1.00 & 0.65 & 0.60 & 0.66 & 0.67 & 0.00 \\
\hline Custo de implantação & 1.00 & 0.76 & 0.75 & 0.80 & 0.79 & 0.00 \\
\hline Custo operacional (vazamentos) & 1.00 & 0.39 & 0.39 & 0.44 & 0.33 & 0.00 \\
\hline Satisfação (tarifa) & 0.41 & 0.51 & 0.34 & 0.53 & 0.51 & 0.33 \\
\hline
\end{tabular}

Além da técnica AHP, outras duas técnicas de análise multicritério foram utilizadas para determinar as melhores alternativas de dimensionamento da rede. A primeira delas, o método Electre II, permite a escolha da alternativa preferida pela maioria dos critérios de acordo com seus respectivos pesos, de forma que não seja ultrapassado um nível de descontentamento do decisor (FIGUEIRA et al., 2016). Nesse caso, são estabelecidas duas estruturas de preferência para a análise das matrizes de concordância e discordância (Tabela 8): uma forte, onde a concordância deve ser superior a 0.7 e a discordância inferior à 0.2, e uma fraca, onde os níveis de tolerância são maiores, compreendendo alternativas com valores de concordância superior a 0.5 e discordância menor que 0.3 .

Tabela 8 - Matrizes de concordância e discordância do método Electre II

\begin{tabular}{|l|l|l|l|l|l|}
\hline \multicolumn{7}{|c|}{ Matriz de Concordância } \\
\hline 0.00 & 0.82 & 0.89 & 0.82 & 0.82 & 0.89 \\
\hline 0.00 & 0.00 & 0.89 & 0.00 & 0.00 & 0.98 \\
\hline 0.00 & 0.00 & 0.00 & 0.00 & 0.55 & 0.98 \\
\hline 0.00 & 0.98 & 0.95 & 0.00 & 0.88 & 0.98 \\
\hline 0.00 & 0.60 & 0.00 & 0.00 & 0.00 & 0.98 \\
\hline 0.00 & 0.00 & 0.00 & 0.00 & 0.00 & 0.00 \\
\hline
\end{tabular}

\begin{tabular}{|c|c|c|c|c|c|}
\hline \multicolumn{7}{|c|}{ Matriz de Discordância } \\
\hline 0.00 & $5.2 \mathrm{E}+07$ & $5.2 \mathrm{E}+07$ & $5.2 \mathrm{E}+07$ & $5.2 \mathrm{E}+07$ & $5.2 \mathrm{E}+07$ \\
\hline 0.61 & 0.00 & 0.81 & 0.53 & 0.22 & 0.28 \\
\hline 0.61 & 0.32 & 0.00 & 0.35 & 0.32 & 0.27 \\
\hline 0.56 & 0.02 & 0.56 & 0.00 & 0.08 & 0.30 \\
\hline 0.67 & 0.06 & 0.66 & 0.30 & 0.00 & 0.22 \\
\hline 1.00 & 0.76 & 0.75 & 0.80 & 0.79 & 0.00 \\
\hline
\end{tabular}

Dessa forma, por meio das classificações progressivas e regressivas apresentadas nas Fig. 5 e 6 , chega-se à classificação final das alternativas. Ressalta-se que a Alternativa 1, por não apresen-

tar relações com as demais, apresentou um alto índice de inconsistência, levando-a à pior classificação após a correção dos valores de ordenação, devido à incerteza sobre sua real classificação. 

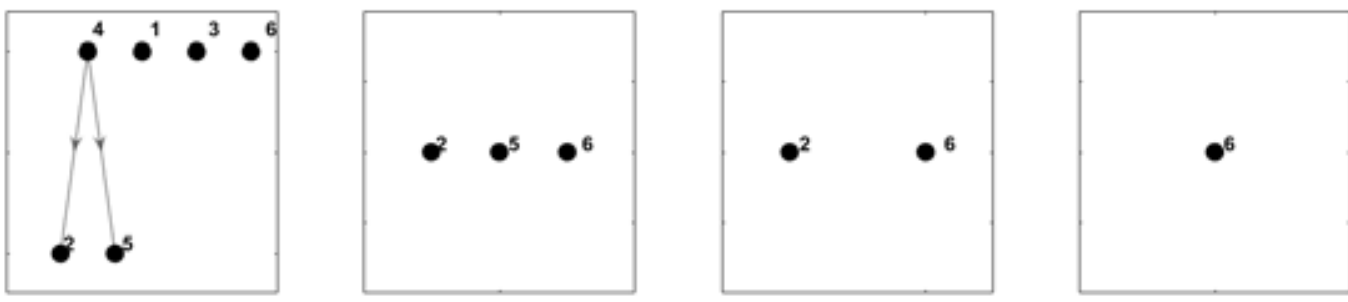

(a)
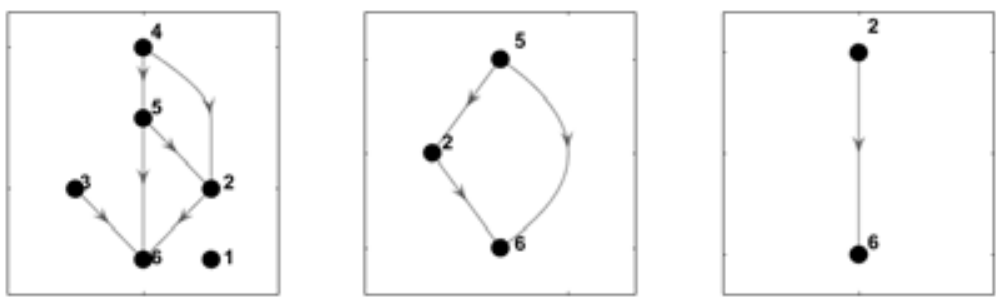

(b)
Figura 5 - Classificação progressiva: a) Estruturação forte; b) Estruturação fraca
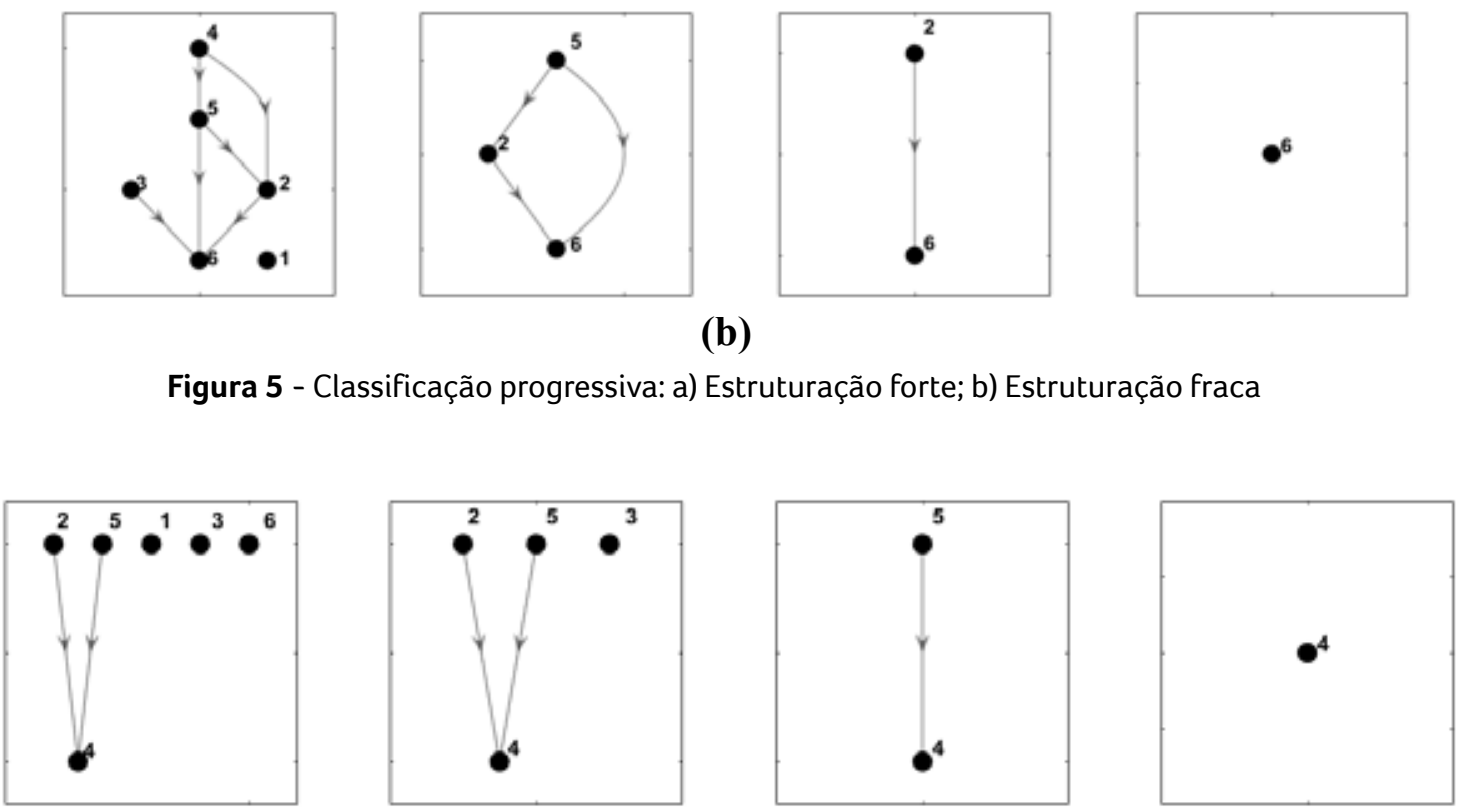
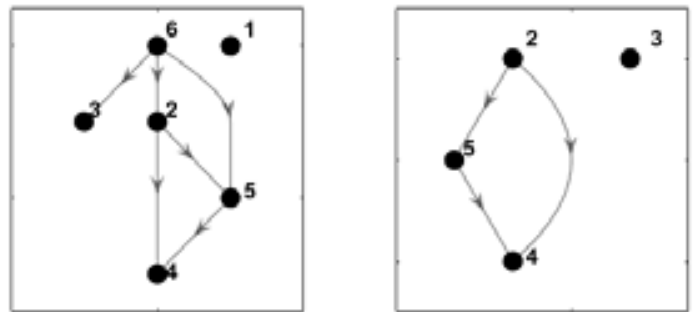

(a)

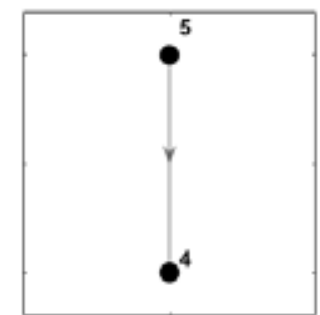

(b)

Figura 6 - Classificação regressiva: a) Estruturação forte; b) Estruturação fraca

O último método aplicado, o Promethee, assim como o Electre, também realiza a comparação par a par entre as alternativas disponíveis. Entretanto, nesse método as diferenças entre os valores de cada critério para duas alternativas distintas são consideradas, mesmo que estas sejam pequenas (BRANS E MARESCHAL, 2005). Dessa forma, cria-se a matriz de preferências apresentada na Tabela 9, em que a somatória dos valores das linhas representa o fluxo positivo da alternativa, e a somatória dos valores das colunas, o fluxo negativo. Assim, fazendo-se o balanço, é possível encontrar as alternativas com melhores fluxos e classificá-las. 
Tabela 9 - Matriz de preferências do método Promethee

\begin{tabular}{|c|c|c|c|c|c|c|c|}
\hline Alternativa & 1 & 2 & 3 & 4 & 5 & 6 & $\begin{array}{c}\text { Fluxo } \\
\text { Positivo }\end{array}$ \\
\hline 1 & 0.00 & 0.07 & 0.07 & 0.06 & 0.07 & 0.15 & 0.420 \\
\hline 2 & 0.01 & 0.00 & 0.01 & 0.00 & 0.00 & 0.08 & $3.9 \mathrm{E}+05$ \\
\hline 3 & 0.01 & 0.00 & 0.00 & 0.00 & 0.00 & 0.08 & $3.9 E+05$ \\
\hline 4 & 0.02 & 0.01 & 0.02 & 0.00 & 0.01 & 0.09 & $3.9 E+05$ \\
\hline 5 & 0.02 & 0.00 & 0.01 & 0.00 & 0.00 & 0.08 & $3.9 E+05$ \\
\hline 6 & 0.01 & 0.00 & 0.01 & 0.00 & 0.00 & 0.00 & $3.9 E+05$ \\
\hline $\begin{array}{c}\text { Fluxo } \\
\text { Negativo }\end{array}$ & $1.9 E+06$ & 0.090 & 0.091 & 0.064 & 0.097 & 0.511 & - \\
\hline Fluxo Total & $-1.93888627 E+06$ & $3.87777342 E+05$ & $3.87777357 E+05$ & $3.87777409 E+05$ & $3.87777339 E+05$ & $3.87776826 \mathrm{E}+05$ & - \\
\hline
\end{tabular}

A classificação das alternativas obtida por meio de cada um dos métodos utilizados é apresentada na Tabela 10. Observa-se a concordância entre os métodos, sendo a Alternativa 4 a melhor opção, coincidindo com a alternativa de menor custo.

Tabela 10 - Classificação das alternativas para cada um dos métodos utilizados

\begin{tabular}{|c|c|c|c|c|c|c|c|}
\hline Alternativa / Método & $\mathbf{1}$ & $\mathbf{2}$ & $\mathbf{3}$ & $\mathbf{4}$ & $\mathbf{5}$ & $\mathbf{6}$ \\
\hline AHP & 6 & 3 & 2 & 1 & 4 & 5 \\
\hline Electre II & 6 & 3 & 4 & 1 & 2 & 5 \\
\hline Promethee & 6 & 3 & 2 & 1 & 4 & 5 \\
\hline
\end{tabular}

\section{CONSIDERAÇÕES FINAIS}

Atualmente, diversos trabalhos têm focado no dimensionamento de redes de abastecimento de água apenas em seu custo de implantação. Entretanto, esse conceito não leva em conta outros fatores importantes, e, em longo prazo, essa alternativa pode se mostrar inviável, gerando maiores gastos ao longo de sua vida útil. Dessa forma, este trabalho criou um mapa cognitivo para identificar os principais critérios a serem avaliados no dimensionamento de uma rede de abastecimento de água. Em seguida, foram calculados os pesos de cada um desses critérios e suas funções de valor, adotando-se as técnicas Delphi e AHP em conjunto. Por fim, três técnicas de avaliação multicritério foram utilizadas para a resolução do estudo de caso apresentado: AHP, Electre II e Promethee. Observou-se uma boa concordância entre os métodos, demonstrando sua aplicabilidade nesse tipo de problema. No estudo de caso apresentado, de- vido aos pesos e às funções de valor adotadas, a melhor alternativa coincidiu com a de menor custo. Entretanto esse resultado é dependente do julgamento dos decisores ao determinarem os pesos dos critérios e suas funções de valor, sendo o resultado final o reflexo dos interesses prioritários de cada sistema.

\section{REFERÊNCIAS}

NBR, ABNT. 12218-Projeto de rede de distribuição de água para abastecimento público. Associação Brasileira de normas Técnicas-ABNT. Rio de Janeiro, 1994.

ACKERMANN, Fran; EDEN, Colin; CROPPER, Steve. Getting started with cognitive mapping. Banxia Software, 1992.

ALPEROVITS, Elyahu; SHAMIR, Uri. Design of optimal water distribution systems. Water resources research, v. 13, n. 6, p. 885-900, 1977.

AMERICAN WATER WORKS ASSOCIATION et al.. Effects of Water Age on Distribution System Water Quality. American Water Works Association: Denver, CO, USA, p. 19, 2002. 
BRANS, Jean-Pierre; MARESCHAL, Bertrand. PROMETHEE methods. In: Multiple criteria decision analysis: state of the art surveys. Springer, New York, NY, 2005. p. 163-186.

BRIOZO, Rodrigo Amancio; MUSETTI, Marcel Andreotti. Método multicritério de tomada de decisão: aplicação ao caso da localização espacial de uma Unidade de Pronto Atendimento-UPA $24 \mathrm{~h}$. Gestão e Produção, v. 22, n. 4, p. 805-819, 2015.

CUNHA, Maria da Conceição; SOUSA, Joaquim. Hydraulic infrastructures design using simulated annealing. Journal of Infrastructure Systems, v. 7, n. 1, p. 32-39, 2001

DALKEY, Norman; HELMER, Olaf. An experimental application of the Delphi method to the use of experts. Management science, $v$. 9, n. 3, p. 458-467, 1963.

DANDY, G. C. et al.. Optimum design and operation of pumped water distribution systems. In: 1994 International Conference on Hydraulics in Civil Engineering:'Hydraulics Working with the Environment'; Preprints of Papers. Institution of Engineers, Australia, 1994. p. 149.

FARMANI, Raziyeh; WALTERS, Godfrey; SAVIC, Dragan. Evolutionary multi-objective optimization of the design and operation of water distribution network: total cost vs. reliability vs. water quality. Journal of Hydroinformatics, v. 8, n. 3, p. 165-179, 2006.

FIGUEIRA, José Rui; MOUSSEAU, Vincent; ROY, Bernard. ELECTRE methods. In: Multiple Criteria Decision Analysis. Springer, New York, NY, 2016. p. 155-185.

GOMES, Ricardo; SÁ MARQQUES, Alfeu; SOUSA, Joaquim. Estimation of the benefits yielded by pressure management in water distribution systems. Urban Water Journal, v. 8, n. 2, p. 65-77, 2011.

MAIER, Holger R. et al.. Ant colony optimization for design of water distribution systems. Journal of water resources planning and management, v. 129, n. 3, p. 200-209, 2003.

MARCHIORI, Isabela Niedo; MEIRELLES, Gustavo; BRENTAN, Bruno Melo; LUVIZOTTO JR, Edevar, Izquierdo, Joaquin. "Avaliação de funções de penalização para o dimensionamento otimizado de redes de distribuição de água”. In: Congresso de métodos numéricos em Ingniería, Valencia, Espanha, 2017. Anais...
MEIRELLES, Gustavo; BRENTAN, Bruno Melo; LUVIZOTTO JR, Edevar. Optimal design of water supply networks using an energy recovery approach. Renewable Energy, v. 117, p. 404-413, 2018.

MONTALVO, Idel; IZQUIERDO, Joaquin, PEREZ-GARCIA, Rafel; HERRERA, Manuel. Water Distribution System Computer Aided Design by Agent Swarm Optimization. Computer Aided Civil and Infrastructure Engineering, v. 29, n. 6, p. 433-448, 2014.

MOOSAVIAN, Naser; ROODSARI, Babak Kasaee. Soccer league competition algorithm: A novel meta-heuristic algorithm for optimal design of water distribution networks. Swarm and Evolutionary Computation, v. 17, p. 14-24, 2014.

OSTFELD, Avi; OLIKER, Nurit; SALOMONS, Elad. Multiobjective optimization for least cost design and resiliency of water distribution systems. Journal of Water Resources Planning and Management, v. 140, n. 12, p. 04014037, 2013.

RIBEIRO DOS SANTOS, Paloma; GUTIERREZ CURO, Rocio Soledad; NEYRA BELDERRAIN, Mischel Carmen. Aplicação do mapa cognitivo a um problema de decisão do setor aeroespacial de defesa do Brasil. Journal of Aerospace Technology and Management, v. 3, n. 2, 2011.

ROSSMAN, Lewis A. et al.. EPANET 2: users manual. 2000.

SAATY, Thomas L. Método de análise hierárquica. Livro, São Paulo, Editora Makron, 1991.

SIMPSON, A.; MURPHY, L.; DANDY, G. Pipe network optimisation using genetic algorithms. In: Proceedings, Specialty Conference of Water Resources Planning and Management Division, ASCE, Seattle, Washington, EUA, 392-395, 1993. Anais...

SWAMEE, Prabhata K.; SHARMA, Ashok K. Design of water supply pipe networks. John Wiley \& Sons, 2008.

TODINI, Ezio. Looped water distribution networks design using a resilience index based heuristic approach. Urban water, v. 2, n. 2, p. 115-122, 2000. 